\title{
Profiling of glycosphingolipids with SCDase digestion and HPLC-FLD-MS
}

\author{
Radhika Chakraberty, Bela Reiz, Christopher W. Cairo* \\ Department of Chemistry, University of Alberta, Edmonton, AB T6G 2G2, \\ CANADA \\ *Corresponding author. Tel.: 780492 0377; fax: 780492 8231; e-mail: \\ ccairo@ualberta.ca
}




\section{Abstract}

Lipid components of cells and tissues feature a large diversity of structures that present a challenging problem for molecular analysis. Glycolipids from mammalian cells contain glycosphingolipids (GSLs) as their major glycolipid component, and these structures vary in the identity of the glycan headgroup as well as the structure of the fatty acid and sphingosine (Sph) tails. Analysis of intact GSLs is challenging due to the low abundance of these species. Here, we develop a new strategy for the analysis of lyso-GSL ( $l$-GSL), GSL that retain linkage of the glycan headgroup with the Sph base. The analysis begins with digestion of a GSL sample with sphingolipid ceramide $N$-deacylase (SCDase), followed by labelling with an amine-reactive fluorophore. The sample was then analyzed by HPLC-FLD-MS and quantitated by addition of an external standard. This method was compared analysis of GSL glycans after cleavage by an Endoglycoceramidase (EGCase) enzyme and labeling with a fluorophore (2-anthranilic acid, 2AA). The two methods are complementary, with EGCase providing improved signal (due to fewer species) and SCDase providing analysis of lyso-GSL. Importantly the SCDase method provides Sph composition of GSL species. We demonstrate the method on cultured human cells (Jurkat T cells) and tissue homogenate (porcine brain). 


\section{Introduction}

Glycosphingolipids (GSLs) are the most common form of glycolipids found in vertebrates. Structurally they bear a carbohydrate headgroup attached to a ceramide (Cer) moiety. The ceramide lipid contains a sphingosine (Sph) base with a fatty acid chain linked via an amide bond.[1, 2] GSLs are ubiquitous in cells and tissues; however, their amounts vary.[3] Neuronal cells have the highest abundance of GSLs, largely composed of gangliosides.[4] Gangliosides are a sub-class of GSLs characterized by the presence of sialic acid (neuraminic acid) residues in the glycan headgroup. Gangliosides function as key players in cell proliferation, cell signaling, and cell-cell interactions.[5] Additionally, these complex biomolecules have been associated with a variety of neurodegenerative conditions such as Alzheimer's [6-8], Huntington's [9-11], and lysosomal storage diseases such as Tay Sach's[12] and Gaucher's[13] diseases. Thus, there is an increasing need for the precise and accurate analysis of GSLs to understand their role in health and disease.

GSL and ganglioside analysis has been an active area of research for decades, the most common methods for analysis are thin layer chromatography (TLC) [14, 15] and mass spectrometry (MS).[16] Although these methods have been used extensively, it remains difficult to obtain quantification or insight into complex structural features of GSLs.[14] When used alone, MS techniques such as ESI-MS[17, 18] or MALDI[19, 20] suffer from inherent drawbacks like ionization suppression and scarcity of suitable internal standards for precise quantification.[21] Thus, MS in combination with LC has been a preferred alternative for quantitative analyses.[21, 22] Existing LC and MS methods typically analyze GSLs after cleavage of the glycan or as fully intact molecules.[23-25] Therefore, many studies neglect analysis of the lipid moiety owing to the complexity of these structures.

The lipid moiety of GSLs is inherently complex due to variability in the saturation and length of both the Sph base and fatty acid. The ceramide portion of GSLs is considered to have a functional role, and variability in the fatty acid chains of Cer has been implicated in disease.[26] The role of Sph bases has not been studied extensively, despite reports showing differential expression of $\mathrm{d} 18: 1$ and $\mathrm{d} 20: 1$ Sph bases in the brain.[27] GSLs 
which have lost the N-acyl fatty acid are known as lyso-GSLs (l-GSLs) and are implicated in Gaucher's disease,[28] Krabbe's disease,[29] and certain cancers.[30] There is a lack of general methods for the analysis of $l$-GSL in complex biological samples.

In the present study, we used two modes of enzymatic digestion of GSL to provide complementary analysis of glycans and $l$-GSL. Traditional chemical methods of GSL degradation such as alkaline hydrolysis,[31] ozonolysis[32] or osmium-catalyzed periodate oxidation[33] produce low yields and require long reaction times and cumbersome purification. Here, we employed Endoglycoceramidase (EGCase) and Sphingolipid ceramide $N$-deacylase (SCDase) enzymes to digest GSL and analyze the resulting products. EGCase catalyzes the cleavage of the ceramide lipid backbone, liberating free oligosaccharides from GSLs, $[34,35]$ while SCDase cleaves the fatty acid chain of the ceramide moiety to produce $l$-GSLs bearing a free amine (Figure 1). [36] We develop a protocol here to label the degradation products of each enzyme with the fluorophores 2-anthranilic acid (2-AA) [34] and Rapifluor-MS,[37] respectively, followed by fluorescent LC-MS analysis. Fluorescent LC-MS (HPLC-FLD-MS) has the advantage of improved sensitivity and reliable quantification using fluorescently labeled standards, which is not possible with MS-only techniques.[38] Our objective in this study was to extend the advantages of enzymatic GSL degradation to HPLC-FLD-MS using these two complimentary approaches which, in the case of SCDase, maintain the linkage of the glycan headgroup and Sph chain of GSLs. We utilized both methods to profile and quantify GSLs in a mammalian T cell line (Jurkat) and porcine brain tissues. 


\section{Materials and methods}

All reagents were purchased from Sigma-Aldrich (Oakville, Ontario, Canada), except for the following: GSL standards LacCer, GM3, GM1, and GD3 were purchased from Avanti Polar Lipids Inc. (Alabaster, Alabama, USA); EGCase I genes from GenScript (Piscataway, NJ, USA); Accucore-150-Amide-HILIC, $2.6 \mu \mathrm{m}, 2.1 \times 150 \mathrm{~mm}$ from Thermo Fisher Scientific (Mississauga, Ontario, Canada), Amicon Ultra-centrifugation filters from Thermo Fisher Scientific (Mississauga, Ontario, Canada), and SepPak C18 cartridge from Waters Corporation (Milford, MA, USA). Porcine brains were obtained from the Swine Research and Technology Centre at the University of Alberta.

\subsection{Jurkat $T$ cell culture}

Jurkat T cells (Clone E6-1, ATCC TIB-152) were purchased from ATCC (Mannassas, VA). Cells were cultured in RPMI 1640 media containing fetal bovine serum (FBS) $(10 \%, \mathrm{v} / \mathrm{v})$ and penicillin $\left(10\right.$ units $\left.\mathrm{mL}^{-1}\right)$ in a humidified incubator with $5 \% \mathrm{CO}_{2}$ at $37{ }^{\circ} \mathrm{C}$. All experiments were performed with cells in passage numbers P3-P5.

\subsection{Preparation of Porcine brain homogenates}

A sample of brain tissue was cut from a full porcine brain of a juvenile pig. This was homogenized in a solvent containing $4 \mathrm{~mL}$ methanol, $2 \mathrm{~mL}$ chloroform, and $1.5 \mathrm{~mL}$ water, in the ratio $1: 5(\mathrm{w} / \mathrm{v})$ using a mechanical homogenizer. Aliquots of homogenates were stored at $-80^{\circ} \mathrm{C}$.

\subsection{Endoglycoceramidase (EGCase) expression and purification}

Expression and purification of EGCase was performed based on the reports of Albrecht et al.,[35] where a gene encoding recombinant EGCase I was identified and synthesized in a pET30 vector (Genscript). Protein expression was performed by culturing E. coli carrying the pET30 vector overnight, followed by 1:100 dilution into 1 litre of LB medium supplemented with $25 \mathrm{mg} / \mathrm{mL}$ Kanamycin. The culture was grown until OD reached $\sim 0.8$ at $37{ }^{\circ} \mathrm{C}$, followed by IPTG induction at $0.1 \mathrm{mM}$ final concentration by shaking at $16^{\circ} \mathrm{C}$ for $18-20 \mathrm{~h}$. Cells were then centrifuged, followed by re-suspension in $45 \mathrm{~mL}$ of $300 \mathrm{mM} \mathrm{NaCl}$ buffer (pH 7.5) containing $0.1 \%$ Triton X-100, $10 \mathrm{mM}$ imidazole, and $50 \mathrm{mM}$ Tris-Cl. Finally, cells were lysed using a cell disruptor. Prior to purification on an AKTA Prime Plus FPLC instrument, centrifugation was 
performed to remove cell debris. For protein purification, a Ni-NTA superflow column (2 $\mathrm{mL})$ was prepared. The elution buffer used was Tris- $\mathrm{HCl}(300 \mathrm{mM} \mathrm{NaCl}, 250 \mathrm{mM}$ imidazole, $50 \mathrm{mM}$ Tris-Cl, $\mathrm{pH}$ 7.5). All fractions were pooled and concentrated using molecular weight cut-off ultracentrifugation filters (30 kDa MWCO). Protein concentration was measured at $280 \mathrm{~nm}$ and protein was stored at $4{ }^{\circ} \mathrm{C}$.

\subsection{Testing EGCase activity}

The activity of EGCase was tested qualitatively on a TLC plate using ganglioside substrate GM3. GM3 substrate and a reaction mixture of GM3+EGCase were spotted on a TLC plate that was developed with a solvent system of acetic acid: $n$-butanol:0.25\% $\mathrm{CaCl}_{2}(1: 2: 1, \mathrm{v} / \mathrm{v} / \mathrm{v})$ and stained with Orcinol stain to visualize GSL-glycans. Specific activity was measured from a calibration curve of serial dilutions of 2-AA labeled 3'sialyllactose (3'-SL). One unit of EGCase I was defined as the amount of enzyme that hydrolyzed $1 \mu \mathrm{mol}$ of GM3 per min at $37{ }^{\circ} \mathrm{C}$.

\subsection{Sphingolipid Ceramide $\boldsymbol{N}$-deacylase (SCDase) Expression and Purification}

Expression and purification of $S$. alga G8 sphingolipid ceramide $N$-deacylase (SA_SCD) was performed following the report of Han et al.[36] The gene encoding recombinant SA_SCD was synthesized in a pET23b vector (Genscript). For protein expression, E. coli carrying the pET23b vector were cultured overnight, followed by 1:100 dilution into $1 \mathrm{~L}$ of $\mathrm{LB}$ medium containing $100 \mathrm{mg} / \mathrm{mL}$ Ampicillin and $35 \mathrm{mg} / \mathrm{mL}$ chloramphenicol. The culture was grown to $\sim 0.8 \mathrm{OD}_{600}$ at $37{ }^{\circ} \mathrm{C}$, followed by IPTG induction at a final concentration of $0.1 \mathrm{mM}$ by shaking at $16^{\circ} \mathrm{C}$ for $18-20 \mathrm{~h}$. Cells were then centrifuged, followed by re-suspension in $40 \mathrm{~mL}$ of $300 \mathrm{mM} \mathrm{NaCl}$ buffer ( $\mathrm{pH}$ 7.5) containing $0.1 \%$ Triton X-100, $10 \mathrm{mM}$ imidazole, and $50 \mathrm{mM}$ Tris-Cl. Finally, cells were lysed by using a cell disruptor. Prior to purification of SCDase, centrifugation was performed to remove any cell debris. For protein purification, a Ni-NTA superflow column $(2 \mathrm{~mL})$ was prepared. Elution was performed in 50 fractions $(1 \mathrm{~mL}$ each; 300 $\mathrm{mM} \mathrm{NaCl}, 250 \mathrm{mM}$ imidazole, $50 \mathrm{mM}$ Tris-Cl, $\mathrm{pH}$ 7.5). Fractions were pooled and concentrated using molecular weight cut-off ultracentrifugation filters from Amicon (50 $\mathrm{kDa} \mathrm{MWCO}$ ). The purified and concentrated protein was stored at $4{ }^{\circ} \mathrm{C}$.

\subsection{Testing SCDase Activity}


The activity of SCDase was tested on a TLC plate using the ganglioside substrate GM3. The GM3 substrate and the reaction mixture of GM3 + SCDase were spotted on a TLC plate that was developed using a solvent system containing acetic acid: $n$ butanol:0.25\% $\mathrm{CaCl}_{2}(1: 2: 1, \mathrm{v} / \mathrm{v} / \mathrm{v})$ and stained with Orcinol stain to visualize $l$-GSL spots. Specific activity was measured from a fluorescence intensity vs time plot of a mixture of RapiFluor-MS labeled $l$-GM3 and $l$-GM1, generated as a result of SCDase digestion at $37{ }^{\circ} \mathrm{C}$ for $14 \mathrm{~h}$. One unit of SCDase was defined as the amount of enzyme that hydrolyzes $1 \mu \mathrm{mol}$ of GM3 or GM1 per minute at $37^{\circ} \mathrm{C}$.

\subsection{Extraction and purification of gangliosides from Jurkat $T$ cells and porcine}

\section{brain homogenate}

Ganglioside extraction and purification was performed based on the reports of Schnaar and co-workers.[39] A sample of $10 \mu \mathrm{L}$ brain homogenate or cell lysate containing $\sim 1 \mathrm{x}$ $10^{6}$ cells were diluted with ice cold water $(4 \mathrm{~mL} / \mathrm{g}$ based on wet weight of sample). After vigorous homogenization, methanol and chloroform were added to make the final chloroform:methanol:water ratio 4:8:3 (v/v/v). This mixture was subjected to centrifugation at $1500 \mathrm{RPM}$ for $15 \mathrm{~min}$. The upper phase was recovered, the volume recorded carefully, and it was diluted with 0.173 volumes of water. After mixing, the suspension was subjected to centrifugation again at 1500 RPM for $15 \mathrm{~min}$. The upper phase containing $\sim 80 \%$ of the total volume was recovered and transferred to a fresh tube. The sample was purified on a Waters SepPak C18 cartridge, evaporated to dryness under nitrogen gas, and re-dissolved in methanol at a volume of $100 \mu \mathrm{L} / \mathrm{mg}$ of dried extract.

\subsection{EGCase I digestion}

The final methanol extract of crude GSLs was dried under nitrogen from a $50 \mathrm{mM}$ sodium acetate buffer ( $\mathrm{pH} 5.2$ ) containing $1 \mathrm{mg} \mathrm{mL}^{-1}$ sodium cholate. For commercially available standards, a mixture of $10 \mu \mathrm{L}$ each of standard LacCer, GM3, GM1, and GD3 were taken from original stock solutions of concentration $\sim 1 \mathrm{mg} / \mathrm{mL}$. Then, GSLs were incubated for $18 \mathrm{~h}$ at $37^{\circ} \mathrm{C}$ with $6 \mathrm{mU}$ EGCase to release the corresponding glycans.

\subsection{Fluorescent labeling of glycans}

Glycans liberated after EGCase digestion were labeled with $40 \mu \mathrm{L}$ of fluorescent mixture (30 mg 2-anthranilic acid, $20 \mathrm{mg}$ boric acid, $40 \mathrm{mg}$ sodium acetate $\cdot 3 \mathrm{H}_{2} \mathrm{O}$, and 45 $\mathrm{mg}$ sodium cyanoborohydride in methanol) at $80{ }^{\circ} \mathrm{C}$ for $45 \mathrm{~min}$ after an appropriate 
concentration of internal standard maltose was added. To this, $1 \mathrm{~mL}$ acetonitrile:water (97:3, v/v) was added and purified on a discovery DPA-6S amide-HILIC column, as described.[34] After equilibration of the column with $2 \times 1 \mathrm{~mL}$ of $100 \%$ acetonitrile, samples were loaded. Then, they were washed with 4 x $1 \mathrm{~mL}$ of acetonitrile:water (99:1) and $0.5 \mathrm{~mL}$ of acetonitrile:water (97:3). Finally, $2 \times 0.6 \mathrm{~mL}$ of pure water was used to elute labeled glycans. Fractions were dried under vacuum before LC-FLD-MS analysis.

\subsection{SCDase Digestion}

The final methanol extract of crude GSLs was dried under a stream of nitrogen, followed by re-suspension of the residue in a $25 \mathrm{mM}$ sodium acetate buffer ( $\mathrm{pH} 5.0$ ) containing $1 \mathrm{mg} \mathrm{mL}^{-1}$ sodium cholate. For commercially available standards, a mixture of $10 \mu \mathrm{L}$ each of standard LacCer, GM3, GM1, and GD3 were taken from the original stock solutions of $\sim 1 \mathrm{mg} / \mathrm{mL}$ concentration. Next, the mixture was incubated for $14 \mathrm{~h}$ at $37{ }^{\circ} \mathrm{C}$ with $30 \mathrm{mU}$ SCDase to release the corresponding $l$-GSLs.

\subsection{Fluorescent Labeling of $\boldsymbol{l}$-GSLs}

The SCDase-released $l$-GSLs were labeled with $24 \mu \mathrm{L}$ of RapiFluor-MS (1 mg in 14.5 $\mathrm{mL}$ DMF) at room temperature for $5 \mathrm{~min}$.[40] After dilution of samples with acetonitrile to a final volume of $400 \mu \mathrm{L}$, the labeled $l$-GSL were purified using GlycoWorks HILIC $\mu$-elution plate from Waters. Briefly, the columns were pre-washed with $200 \mu \mathrm{L}$ of water, followed by $200 \mu \mathrm{L}$ of $85 \%$ acetonitrile, and then the samples were loaded on to the columns. Next, the columns were washed with two $600 \mu \mathrm{L}$ volumes of formic acid/water/acetonitrile in a ratio of 1:9:90 (v/v/v). Finally, the $l$-GSLs were eluted with three $30 \mu \mathrm{L}$ volumes of elution buffer containing $200 \mathrm{mM}$ ammonium acetate in $5 \%$ acetonitrile.

\subsection{HPLC-FLD-MS Analysis of 2AA-labeled glycans}

Labeled glycans were analyzed by LC-MS using an Agilent 1200 SL HPLC system and a normal-phase column (Accucore-150-Amide-HILIC, $2.6 \mu \mathrm{m}, 2.1 \times 150 \mathrm{~mm}$, Thermo Fisher). Dried samples were re-solubilized in water:DMF:acetonitrile in a 1:1:2 ratio and $2 \mu \mathrm{L}$ (commercially available standards) and $15 \mu \mathrm{L}$ (cells, brain homogenates and plasma) were injected. The fluorescence detector monitored at an excitation of 320 $\mathrm{nm}$ and an emission of $420 \mathrm{~nm}$. All chromatography was performed at $40^{\circ} \mathrm{C}$. The binary solvent system followed a linear gradient with a flow rate of $0.4 \mathrm{~mL} \mathrm{~min}{ }^{-1}$ (Solvent A: 
$100 \mathrm{mM}$ ammonium formate, $\mathrm{pH}$ 4.45; Solvent B: acetonitrile). Mass spectra were acquired in negative mode using an Agilent 6220 Accurate-Mass TOF HPLC/MS system with a dual spray electrospray ionization source, along with a secondary reference sprayer for a reference mass solution.

2.13 HPLC-FLD-MS Analysis of RapiFluor-labeled l-GSLs on a Mixed Mode Weak Anion Exchange (WAX)-Reverse Phase (RP) GlycanPAC AXR-1 Analytical

\section{Column}

RapiFluor labeled l-GSLs were analyzed by LC-FLD-MS using an Agilent 1200 SL HPLC system and a mixed mode column (Dionex/Thermo Fisher, GlycanPAC AXR-1, $1.9 \mathrm{um}, 2.1 \times 150 \mathrm{~mm}$ ). Samples in $5 \%$ acetonitrile buffer were loaded onto the column in $15 \mu \mathrm{L}$ injections. The fluorescence detector was set to monitor the excitation at $265 \mathrm{~nm}$ and the emission at $465 \mathrm{~nm}$. All chromatography was performed at $50{ }^{\circ} \mathrm{C}$. The binary solvent system followed a linear gradient with a flow rate of $0.4 \mathrm{~mL} \mathrm{~min}^{-1}$ (Solvent A: dd Water; Solvent B: $50 \mathrm{mM}$ ammonium formate, $\mathrm{pH} 4.45$ in 40:60 water/acetonitrile). The flow was split 50:50 between the fluorescence and mass spectrometry (MS) detectors. Accurate mass measurement on an Agilent 6220 Accurate-Mass TOF HPLC/MS system was used to assign and confirm the identity of fluorescent peaks. Mass spectra were acquired in the positive mode using a dual spray electrospray ionization source with the secondary reference sprayer used for a reference mass solution. Data analysis was performed using the Agilent MassHunter Qualitative Analysis software package version B.07.01.

\section{Results \& Discussion}

3.1 EGCase digestion and HPLC-FLD-MS analysis of glycosphingolipids from cultured cells and tissue

Our method for glycan analysis was adapted from the work of Neville et al., where an endoglycosidase enzyme, ceramide glycanase, was used to generate glycans devoid of the ceramide chain. Glycans were then labeled with a sensitive fluorophore, 2-aminobenzoic acid (2AA).[34] We followed the same general approach after making changes to the enzyme, labeling conditions, chromatographic conditions, and providing a strategy for quantitation. We selected Endoglycoceramidase (EGCase) for digestion of the glycans, 
which has broad substrate specificity cleaving both acidic and neutral GSLs with equal efficiency.[35] The temperature and time conditions for enzyme digestion were carefully monitored (Figure S1) and we found that $37^{\circ} \mathrm{C}$ and $18 \mathrm{~h}$ incubation yielded optimal results. Similarly, titration experiments were performed with variable concentrations of 2AA added, addition of $40 \mu \mathrm{L}$ of the fluorophore mixture providing maximum signal (Figure S2). We identified an internal standard, maltose, that was used to quantify the results (Figure S3). The limit of detection of our assay was approximately 2 pmol, in agreement with previous studies (Figure S4).[41]

We applied the optimized EGCase method to profiling GSLs from a mammalian cell line (Jurkat T cells) and porcine brain tissue. GSLs were extracted from cells and tissues using the most widely accepted extraction protocol devised by Svennerholm, involving a mixture of chloroform:methanol:water in the ratio $4: 8: 3$ as the extraction solvent.[42] This protocol yielded optimal results when tested on a mixture of LacCer, GM3, GM1, and GD3 (Figure S5). Moreover, with this extraction protocol cell numbers in the range $0.2-1 \times 10^{6}$ cells or $10 \mu \mathrm{L}$ of brain homogenate $(\sim 2 \mathrm{mg}$ dried tissue, or $0.3-0.5 \mathrm{mg}$ dry GSL) was sufficient to generate sensitive results for quantification. Complete GSL profiles in Jurkat $\mathrm{T}$ cells and porcine brain are shown in Figure 2. The GSL profile in Jurkat T cells showed the presence of LacCer (5.9 min), GM3 (8.2 min), GM2 (9.6 min), GM1 (12.9 $\mathrm{min})$, GD3 (14.3 $\mathrm{min})$, and GD1 (16.2 $\mathrm{min})$. GM3 and GM2 were the dominant glycans, with GM3 taking up over $90 \%$ of the composition. While lipid profiles in mammalian cells, including Jurkat have been reported in the literature, many lack data on the GSL class.[43, 44] Additionally, previous analyses were based on MS only, which does not provide accurate quantitation. Some previous reports on GSL profiling in Jurkat cells using the same assay have identified LacCer, GM3, GM2, GD3, GD1a, and GM1 as the major components. However, these reports required larger amounts of starting material, were qualitative, or provided only relative quantification based on an external standard.[45-47] Neuronal cells are enriched with GSLs, mostly in the form of gangliosides.[14] The GSL profile in porcine brain is exemplary, with an abundance of complex gangliosides such as GM1, GD1a, GD1b, Fuc-GM1, Fuc-GD1, and GT1. Previous reports have yielded results similar to ours, with a few exceptions such as the presence of more complex gangliosides GT3 and GQ1.[48-50] We attributed these 
differences to the fact that ganglioside expression is age dependent, and complex gangliosides are more common with age.[51, 52] Our samples were from juvenile pigs; which may explain the lack of more complex gangliosides in our data. Thus, our EGCase assay provides improved quantitative analysis of GSLs by employing HPLC-FLDMS.[32, 48, 49, 53, 54]

\subsection{SCDase digestion and analysis of lyso-glycosphingolipids by HPLC-FLD-MS}

Taking inspiration from the EGCase assay, we moved on to developing a quantitative method that would provide insight into the structure of the sphingosine base, which is largely overlooked in standard GSL analyses. The enzyme sphingolipid ceramide $\mathrm{N}$ deacylase (SCDase) has been previously used for GSL degradation, to generate 1-GSLs bearing the carbohydrate chain and Sph base.[55, 56] Although this enzyme offers an excellent alternative to common chemical degradation strategies, it is seldom used since it has not been characterized extensively. A study by Withers and co-workers addressed this problem, wherein a new form of the SCDase enzyme (SA_SCD) was characterized and reported to be universally active on all GSLs under favourable temperature and pH.[36] We selected this form of the enzyme to generate l-GSL. Importantly, we expected that $l$-GSLs generated from SCDase digestion could be easily labeled with an amine-reactive fluorophore (Figure 1). We selected RapiFluor MS, a sensitive fluorophore used for glycopeptide analysis that also provides enhancement of MS signal.[37]

Experiments were conducted to scan for optimal temperature and incubation times for SCDase digestion. For temperature conditions, a mixture of GM1 and GM3 standards were subjected to enzymatic digestion at $25,30,37$, and $45{ }^{\circ} \mathrm{C}$ for $24 \mathrm{~h}$, followed by RapiFluor-MS labeling of lyso-products and LC-MS analysis. Evaluation of the combined fluorescence intensity of all $l$-GSLs at each temperature found that $37^{\circ} \mathrm{C}$ gave maximum yields (Figure S6). These results were then used to optimize SCDase concentration that would provide optimal generation of $l$-GSLs. We observed that 25-30

$\mathrm{mU}$ for $14 \mathrm{~h}$ provided optimum cleavage of GSLs by SCDase (Figure S7, S8). Labeling conditions were optimized by conducting titration experiments using a fixed quantity of GM1 standard and subjecting it to SCDase digestion followed by labeling with increasing volumes $(4,8,12,16$, and $24 \mu \mathrm{L})$ of RapiFluor MS (Figure S9). There was a gradual 
increase in response with increasing amounts of RapiFluor addition and the maximum response was observed at $24 \mu \mathrm{L}$. The limit of detection for this assay was $3.0 \mathrm{ng}$ or approximately 2 pmol based on the molecular weight of one form of GM1 [1546.823 g/mol for GM1 (d18:1-18:0)] (Figure S10).

With an optimized GSL SCDase digestion protocol in hand we proceeded to develop a chromatographic strategy. We recognized that a mixture of $l$-GSL would introduce substantial complexity into the chromatograms, and we would require improved separation of $l$-GSLs for such a mixture. Preliminary experiments with C8 and C18 reverse phase columns with a mixture of GM1, GM3, and GD3 standards were unsatisfactory (Figure S11, S12). We found that C8 and C18 media were able to resolve differences in Sph chain length but did not resolve differences in the glycan structure. We next investigated a mixed-mode column that offers RP and weak anion exchange (WAX) modes of separation to resolve these mixtures of amphiphilic l-GSL. The GlycanPac AXR-1 column provided good separation for mixtures of $l$-GSL from GM1, GM3, and GD3 standards (Figure 3). We next required a standard for quantitation of the SCDase assay. In the absence of a commercially available $l$-GSL standard, we sought a standard to determine the specific activity of SCDase. We selected a synthetic compound with features similar to a $l$-GSL, 8-[(2-aminoethyl)thiol)]-1-octyl- $\beta$-D-galactopyranosyl$(1 \rightarrow 4)$ - $\beta$-D-glucopyranoside 1 (Figure S13), bearing an octyl chain and a primary amine available for labeling with RapiFluor MS.[57] Compound 1 was well resolved from all Rapifluor-labelled $l$-GM1, $l$-GM3, and $l$-GD3 products (Figure S14). This standard was used to determine the specific activity of SCDase with a standard GSL mixture over 2, 8, 14, and $24 \mathrm{~h}$. The absolute concentration all forms of $l$-GM3 (d16:1, d17:1, and d18:1) and all forms of $l$-GM1 (d18:1 and d20:1) were calculated from the known concentration of compound 1 added to the reaction mixture.

\subsection{Comparison of EGCase and SCDase GSL profiles in biological samples}

The goal of our study was to establish complementary methods of GSL analysis using two different modes of enzymatic degradation to analyze glycans and $l$-GSLs in a single biological sample. The advantage of this approach is that it could reveal variation in glycan and Sph structures. Thus, we proceeded to test both methods on an identical set of samples to compare their findings. We first ensured consistent recovery of 1-GSLs after 
digestion and post-labeling clean up protocols (Figure S15). Profiling of $l$-GSLs in Jurkat cells required higher cell counts compared to the EGCase assay (1-5 x $10^{7}$ cells). A complete profile of $l$-GSLs from Jurkat cells is shown in Figure 4a. We observed multiple Sph lipids attached to the same glycan (denoted by carbon chain length of the $d$ erythro-sphingosine chain and number of degrees of unsaturation). Major $l$-GSL observed included l-GM3 (d16:1, 10.5 min; d17:1, 13.4; d18:1, $17.3 \mathrm{~min}$; d18:2, 12.5), l-GM1 (d16:1, $10.0 \mathrm{~min}$; d18:1, $16.3 \mathrm{~min}$; d18:2, $11.8 \mathrm{~min}$ ), l-GM2 (d16:1, $10.3 \mathrm{~min}$; d18:1, 16.8 min; d18:2, $12.2 \mathrm{~min}$ ), and $l$-GDla (d16:1, $14.6 \mathrm{~min}$; d18:1, $23.9 \mathrm{~min})$. Acetylated forms of GM3 (6.3 min), GM2 (6.7 $\mathrm{min})$, and GD1a $(8.3 \mathrm{~min})$ bearing the d16:1 chain. The relative standard error (RSE) for three replicate runs was within $20 \%$ for most of the $l$ GSLs, while some ranged between 20 and 30\%. Peak identification and confirmation was carried out by retention time matching (where available) and by determining the elemental composition using accurate mass MS detection. The method was sensitive enough to detect the presence of odd-number chain lengths such as GM3 (d17:1), while not expected to be major forms - odd-number chains are possible for Sph and fatty acids and may be biomarkers of disease.[58, 59] Comparing the results of the EGCase and SCDase assays finds that the two methods agree on the identity of all major GSLs found in Jurkat samples (GM3, GM2, GM1, and GD1a). However, LacCer is not seen in the SCDase data as the enzyme does not cleave neutral GSLs effectively.[36] A direct comparison of the quantitation for the two methods with Jurkat samples is shown in Figure 5a-b (Table S5). While there are variations between the assays, there is good general agreement for the Jurkat samples in both assays (Figure $5 \mathbf{b}, \mathrm{r}^{2}=0.81$ ).

We next tested GSL samples from porcine brains in the SCDase assay, we found that $30 \mu \mathrm{L}$ of brain homogenate ( $\sim \mathrm{mg}$ tissue, or $0.8-1.5 \mathrm{mg}$ dry GSL) was sufficient for analysis. Figure 4c shows a complete $l$-GSL profile from porcine brain comprising of $l$ GM3 (d16:1, 10.0 min; d18:1, $17.2 \mathrm{~min})$, -GM1 (d18:2, $11.8 \mathrm{~min}$; d18:1, $16.8 \mathrm{~min})$, GD1 (d16:1, $14.8 \mathrm{~min}$; d18:1, 22.8 and $23.4 \mathrm{~min}$; d20:1, $36.1 \mathrm{~min}), l-G T 1$ (d18:1, 34.5 min), and l-Fuc-GM1 (d18:1, 15.2 min). Quantitation of signals using compound $\mathbf{1}$ as a standard had a relative standard error (RSE) for three replicate runs within $20 \%$ for the composition of each $l$-GSL, except for $l$-Fuc-GM1 (d18:1), which was $23.5 \%$. The profile shows two peaks assigned as $l$-GD1 (d18:1), which is likely composed of two isomers of 
GD1 (i.e. GD1a and GD1b). Unlike the EGCase method, we could not assign isomers based on chromatographic separation without standards. As seen for Jurkat samples, all major GSLs seen in the EGCase analysis were identified in the SCDase assay (GM3, GM1, Fuc-GM1, and GD1a/b), with the exception of LacCer and GSL in low abundance (GM2, Fuc-GD1; Figure 4d).[36] In the case of porcine brain samples, we saw larger differences between the EGCase and SCDase methods (Figure 5c-d, Table S6) and reduced correlations (Figure 5d, $r^{2}=0.68$ ).

Importantly, in both cases the SCDase assay provides quantitation of Sph composition as it relates to individual glycan structures which cannot be obtained from other methods; for example, GM3 Sph chains were primarily d18:1 in both Jurkat and porcine samples, but d17:1 Sph was only observed in GM3 from Jurkat. Additionally, we observed GM1 d18:2 in porcine brain. We are not aware of previous reports that have tested SCDase on complex tissue samples, with previous work using only standard GSLs.[36, 60, 61] Previous analyses of GSL Sph composition have found a higher prevalence of (d18:1) and (d20:1) in pig and mouse brains, and have not observed d18:2.[62, 63]

\section{Conclusion}

Among the constituents of an organism, lipids show large diversity in structure, making them challenging to analyze.[64] Lipidomic strategies have made significant progress as a platform to study many classes of lipids such as glycerolipids, glycerophospholipids, sphingolipids, and sterols.[65, 66] Glycosphingolipids (GSLs), as a class, are not very well characterized owing to their diversity in structure and challenging amphipathic properties. This study aimed to address these challenges by implementing two complementary methods that link structural information about the glycan component of GSLs with the sphingosine base from a single biological sample. The use of enzymatic degradation strategies with EGCase and SCDase enzymes provided convenient digestion of samples to a free glycan and $l$-GSL, respectively. Fluorophores, 2AA and RapiFluor MS, provided sensitivity for identification and quantification of each GSL component by HPLC-FLD-MS. We believe this is the first application of SCDase for analysis of complex GSL mixtures. We note that our method would benefit from improvements in peak separation of $l$-GSLs or improved MS sensitivity to identify low 
abundance species. Finally, we propose that the methods described here can provide inroads to the development of more complete quantification and analysis of GSL by preserving linkage information between Sph chains and glycan headgroups of GSLs.

\section{Acknowledgements}

The authors would like to thank Waters Corporation for helpful discussions and provision of samples of RapiFluor-MS reagent. We thank Dr. G Daskhan (University of Alberta) for providing a sample of compound 1, and K. McGowan and R.B. Zheng for helpful discussions. This work was supported by a grant from the Natural Sciences and Engineering Research Council of Canada (NSERC RGPIN-2020-04371) and the Canadian Glycomics Network (GlycoNet).

\section{Supplementary data}

The following are available online: Figure S1: Optimization of EGCase digestion conditions; Figure S2: Optimization of 2-AA labeling conditions; Figure S3: Selection of an internal standard for EGCase; Figure S4: Determination of Limit of Detection (LOD) for EGCase; Figure S5: Percent recovery of a standard GSL mixture containing LacCer, GM3, GM1, and GD3 after EGCase digestion; Figure S6: Optimization of temperature and incubation time for SCDase digestion of GSLs; Figure S7: Optimization of SCDase activity; Figure S8: Determination of SCDase activity; Figure S9: Optimization of RapiFluor-MS labeling conditions; Figure S10: Determination of Limit of Detection (LOD) for SCDase; Figure S11: Separation of RapiFluor labeled 1-GM3, 1-GD3, and 1GM1 in a mixture on a Reverse Phase (RP) C18 column; Figure S12: Separation of RapiFluor labeled lyso-GSL on RP-C8; Figure S13: Structure of Compound 1; Figure S14: Compound 1 as an internal standard; Figure S15: Percent recovery of a standard GSL mixture containing GM3, GM1, and GD3; Table S1: Absolute values of concentrations of individual GSLs in Jurkat T-cells by EGCase; Table S2: Absolute values of concentrations of individual GSLs in porcine brain by EGCase; Table S3: Absolute values of concentrations of individual 1-GSLs in Jurkat T cells; Table S4: Absolute values of concentrations of individual 1-GSLs in porcine brain; Table S5: 
Comparison of percent composition of major GSLs in Jurkat $\mathrm{T}$ cells identified independently in the EGCase and SCDase assays; Table S6: Comparison of percent composition of major GSLs in porcine brain identified independently in the EGCase and SCDase assays. 
Figures and Tables

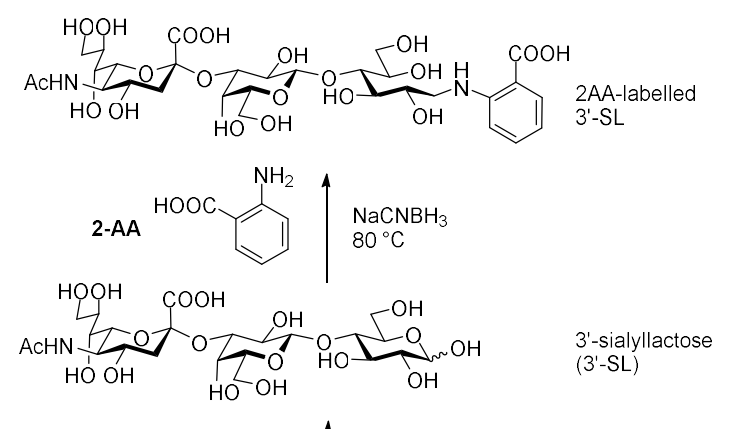

(a) $\uparrow$ EGCase

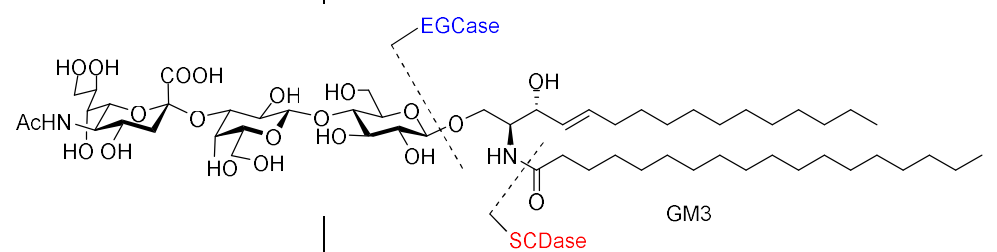

(b) SCDase
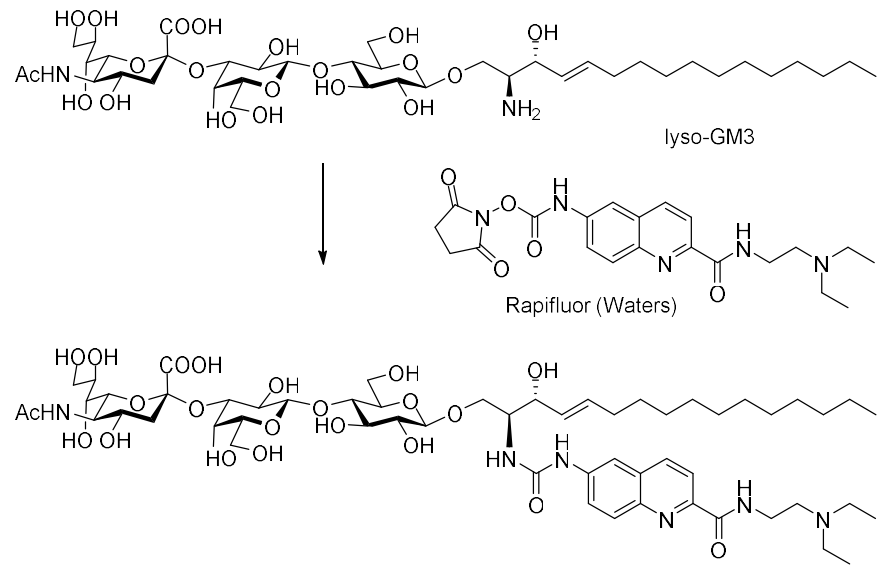

Figure 1: Digestion of GSL using EGCase and SCDase for analysis by LC-MS-FLD 
(a)

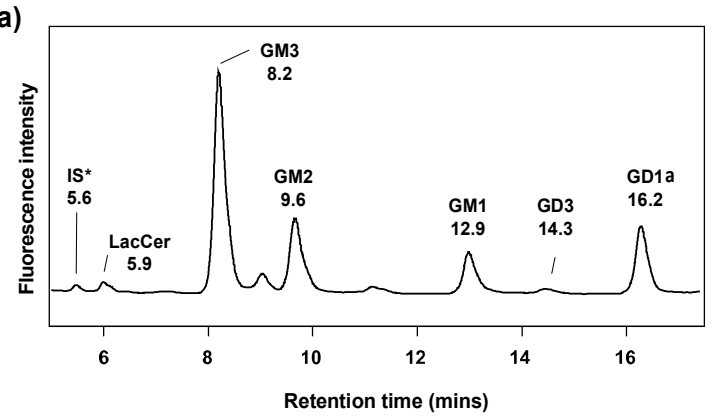

(c)

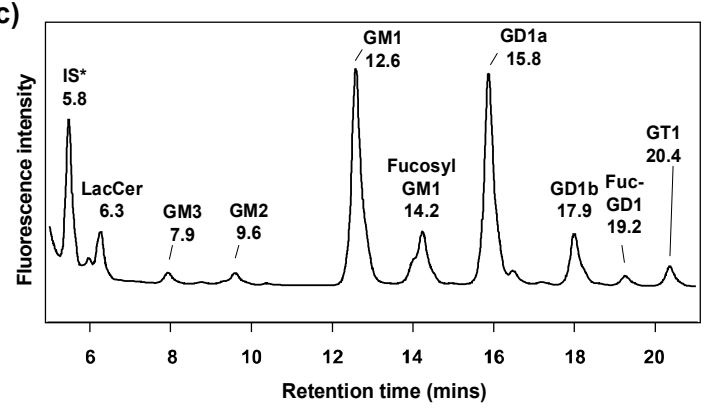

(b)

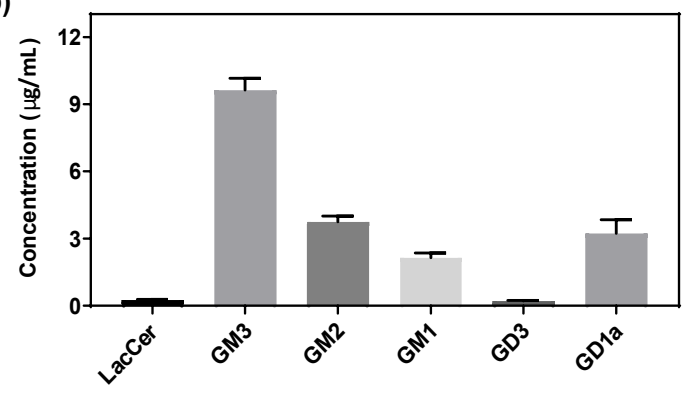

(d)

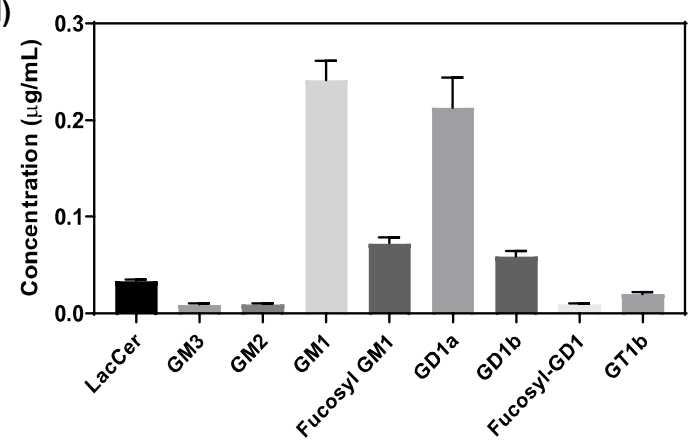

Figure 2. Glycosphingolipid profiling with EGCase digestion. Samples of GSL were digested with EGCase and labelled with 2-AA. GSLs LacCer, GM3, GM2, GM1, GD3, GD1a, GD1b, GT1b, Fuc-GM1, and Fuc-GD1 from (a-b) Jurkat T cells and (c-d) porcine brain are shown. Quantification was performed based on the concentration of internal standard (IS*, maltose). Values represent mean \pm standard error of mean of triplicate measurements, $\mathrm{n}=3$. See Tables $\mathbf{S 1}$ (Jurkat) and $\mathbf{S 2}$ (porcine) for assignments and quantitation. 
(a)

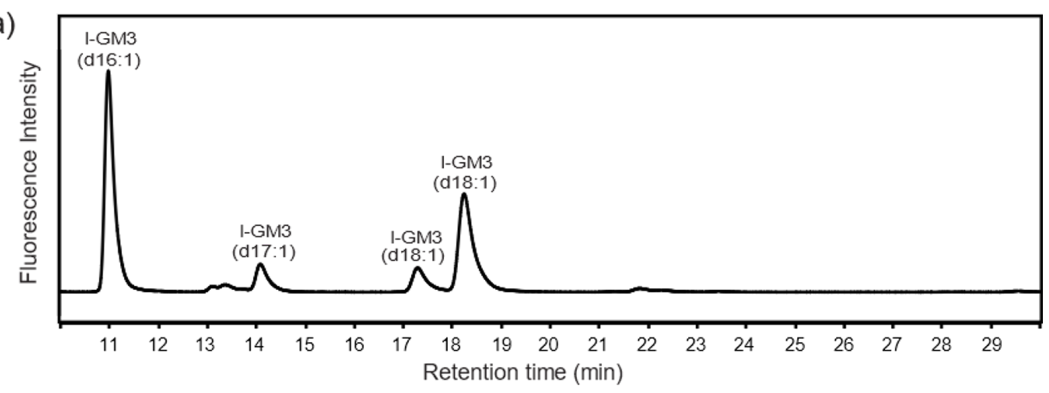

(b)

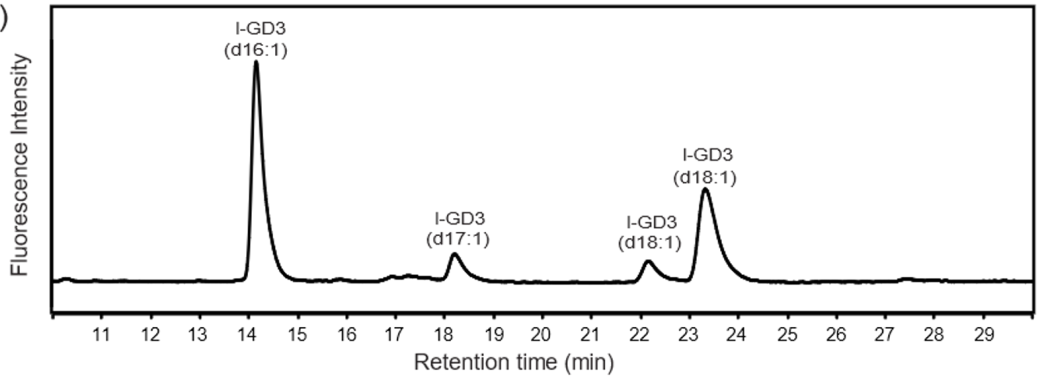

(c)

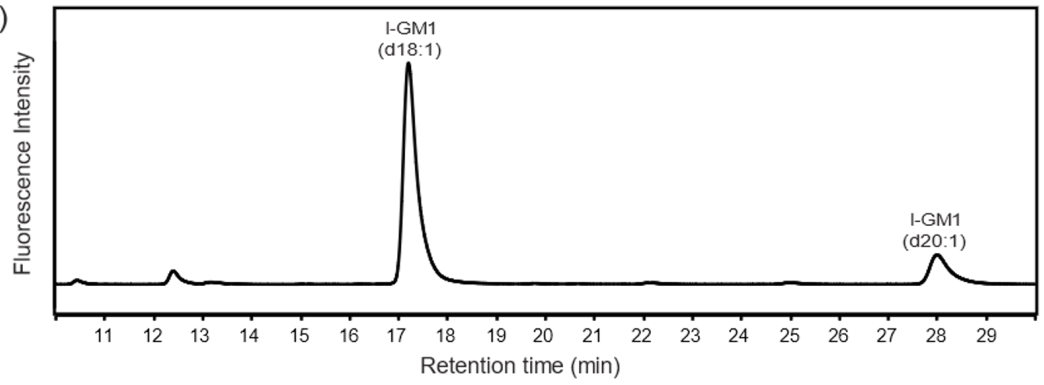

(d)

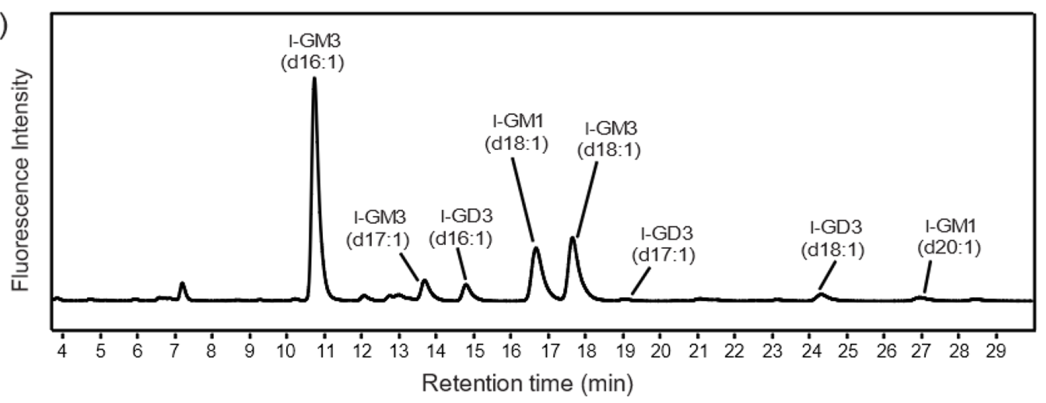

Figure 3. Chromatographic separation of RapiFluor labeled $l$-GSLs on a mixed mode, weak anion exchange-reverse phase (WAX-RP) GlycanPAC AXR-1 column (a) $l$-GM3, (b) $l$-GD3, (c) $l$-GM1, and (d) a mixture of $l$-GM3, $l$-GD3, and $l$-GM1 on the weak anion exchange-reverse phase (WAX-RP) GlycanPAC AXR-1 column. GM3 and GD3, both sourced from bovine milk, show peaks for $l$-GSLs with d16:1, d17:1, and d18:1 Sph bases, while GM1, sourced from porcine brain, shows d18:1 and d20:1 Sph bases. This column showed an improved separation of the peak clusters that were previously seen in the $\mathrm{C} 8$ and $\mathrm{C} 18$ columns. 

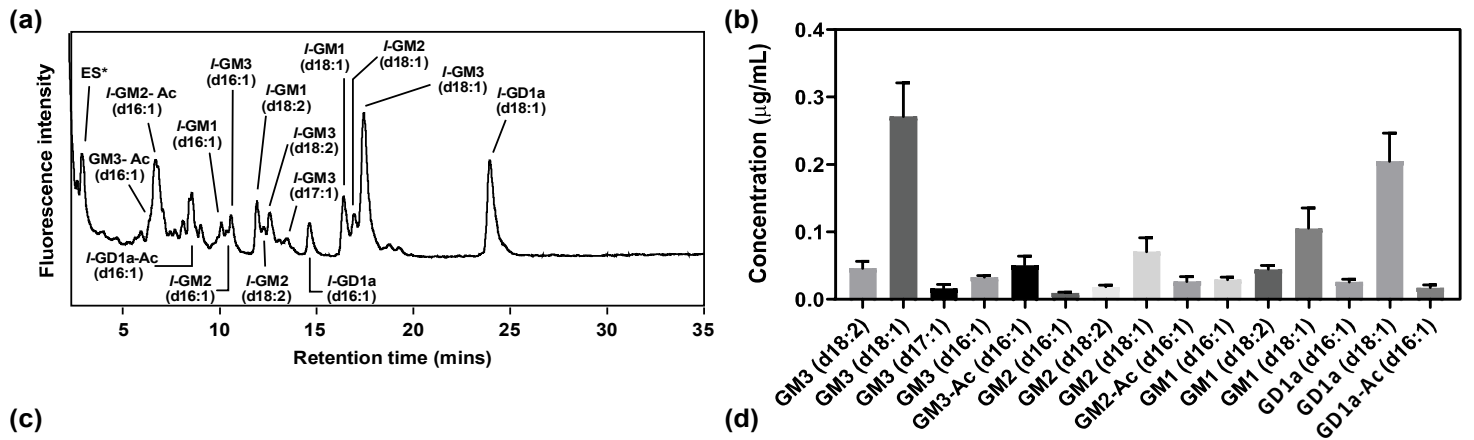

(c)
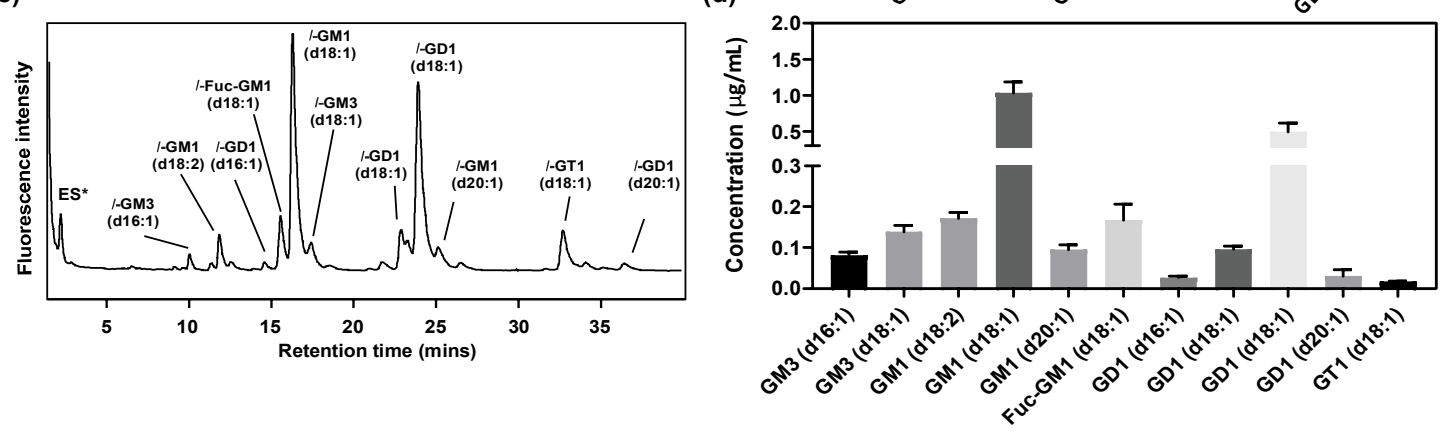

Figure 4. l-GSL profiling with SCDase digestion. Samples of GSL were digested with SCDase and labelled with RapiFluor-MS to generate labeled $l$-GSLs from (a-b) Jurkat T cells and (c-d) porcine brain. Panels (b) and (d) show quantification of the concentration of individual $l$-GSLs. Quantification was performed based on the concentration of external standard (ES*, compound 1) added to the mixture. (Values represent the mean \pm standard error of the mean of triplicate measurements, $n=3$.) See Tables S3 (Jurkat) and S4 (porcine) for assignments and quantitation. 
(a)

(c)
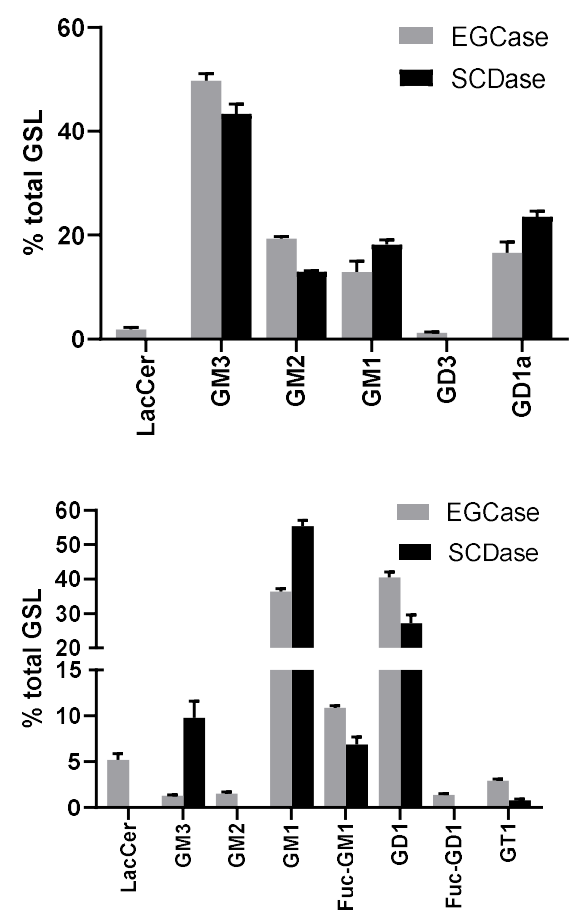

(b)

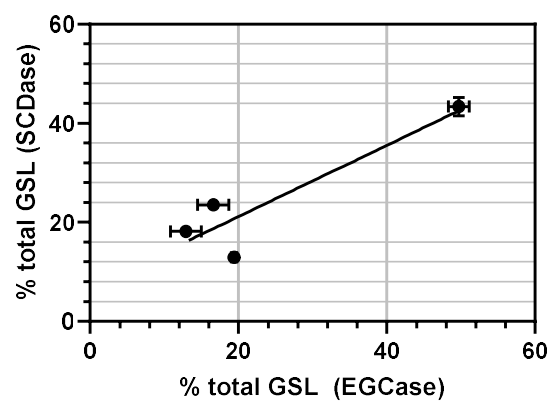

(d)

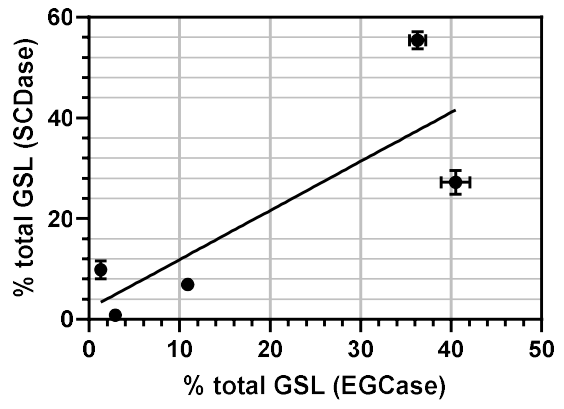

Figure 5. Comparison of relative percentages of GSLs using both methods. Comparison of GSLs LacCer, GM3, GM2, GM1, GD3, and GD1a identified in (a-b) Jurkat $\mathrm{T}$ cells or (c-d) porcine brain from glycan analysis (EGCase method) and $l$-GSL analysis (SCDase method). (a, c) Values represent the mean \pm standard error of the mean of triplicate measurements, $\mathrm{n}=3$. (b, d) See Tables S5 (Jurkat) and S6 (porcine) for values. 


\section{References}

1. van Meer, G.; Voelker, D. R.; Feigenson, G. W., Membrane lipids: where they are and how they behave. Nat Rev Mol Cell Biol 2008, 9, (2), 112-24.

2. Hannun, Y. A.; Obeid, L. M., Sphingolipids and their metabolism in physiology and disease. Nat Rev Mol Cell Biol 2018, 19, (3), 175-191.

3. Merrill, A. H., Jr., Sphingolipid and glycosphingolipid metabolic pathways in the era of sphingolipidomics. Chem Rev 2011, 111, (10), 6387-422.

4. Merrill, A. H., Jr., De novo sphingolipid biosynthesis: a necessary, but dangerous, pathway. J Biol Chem 2002, 277, (29), 25843-6.

5. Groux-Degroote, S.; Guerardel, Y.; Delannoy, P., Gangliosides: Structures, Biosynthesis, Analysis, and Roles in Cancer. Chembiochem 2017, 18, (13), 11461154.

6. Yanagisawa, K., GM1 ganglioside and Alzheimer's disease. Glycoconj $J$ 2015, 32, (3-4), 87-91.

7. Ikeda, K.; Yamaguchi, T.; Fukunaga, S.; Hoshino, M.; Matsuzaki, K., Mechanism of amyloid beta-protein aggregation mediated by GM1 ganglioside clusters. Biochemistry 2011, 50, (29), 6433-40.

8. Hong, S.; Ostaszewski, B. L.; Yang, T.; O'Malley, T. T.; Jin, M.; Yanagisawa, K.; Li, S.; Bartels, T.; Selkoe, D. J., Soluble Abeta oligomers are rapidly sequestered from brain ISF in vivo and bind GM1 ganglioside on cellular membranes. Neuron 2014, 82, (2), 308-19.

9. Imarisio, S.; Carmichael, J.; Korolchuk, V.; Chen, C. W.; Saiki, S.; Rose, C.; Krishna, G.; Davies, J. E.; Ttofi, E.; Underwood, B. R.; Rubinsztein, D. C., Huntington's disease: from pathology and genetics to potential therapies. Biochem $J$ 2008, 412, (2), 191-209.

10. Alpaugh, M.; Galleguillos, D.; Forero, J.; Morales, L. C.; Lackey, S. W.; Kar, P.; Di Pardo, A.; Holt, A.; Kerr, B. J.; Todd, K. G.; Baker, G. B.; Fouad, K.; Sipione, S., Disease-modifying effects of ganglioside GM1 in Huntington's disease models. EMBO Mol Med 2017, 9, (11), 1537-1557.

11. Maglione, V.; Marchi, P.; Di Pardo, A.; Lingrell, S.; Horkey, M.; Tidmarsh, E.; Sipione, S., Impaired ganglioside metabolism in Huntington's disease and neuroprotective role of GM1. J Neurosci 2010, 30, (11), 4072-80.

12. Sango, K.; Yamanaka, S.; Hoffmann, A.; Okuda, Y.; Grinberg, A.; Westphal, H.; McDonald, M. P.; Crawley, J. N.; Sandhoff, K.; Suzuki, K.; Proia, R. L., Mouse models of Tay-Sachs and Sandhoff diseases differ in neurologic phenotype and ganglioside metabolism. Nat Genet 1995, 11, (2), 170-6.

13. Benito, J. M.; Garcia Fernandez, J. M.; Ortiz Mellet, C., Pharmacological chaperone therapy for Gaucher disease: a patent review. Expert Opin Ther Pat 2011, 21, (6), 885-903.

14. In Essentials of Glycobiology, rd; Varki, A.; Cummings, R. D.; Esko, J. D.; Stanley, P.; Hart, G. W.; Aebi, M.; Darvill, A. G.; Kinoshita, T.; Packer, N. H.; Prestegard, J. H.; Schnaar, R. L.; Seeberger, P. H., Eds. Cold Spring Harbor (NY), 2015.

15. Scandroglio, F.; Loberto, N.; Valsecchi, M.; Chigorno, V.; Prinetti, A.; Sonnino, S., Thin layer chromatography of gangliosides. Glycoconj $J$ 2009, 26, (8), 961-73. 
16. El-Aneed, A.; Cohen, A.; Banoub, J., Mass Spectrometry, Review of the Basics: Electrospray, MALDI, and Commonly Used Mass Analyzers. Applied Spectroscopy Reviews 2009, 44, (3), 210-230.

17. Farwanah, H.; Kolter, T.; Sandhoff, K., Mass spectrometric analysis of neutral sphingolipids: methods, applications, and limitations. Biochim Biophys Acta 2011, 1811, (11), 854-60.

18. Olling, A.; Breimer, M. E.; Peltomaa, E.; Samuelsson, B. E.; Ghardashkhani, S., Electrospray ionization and collision-induced dissociation time-of-flight mass spectrometry of neutral glycosphingolipids. Rapid Commun Mass Spectrom 1998, $12,(10), 637-45$.

19. Distler, U.; Hulsewig, M.; Souady, J.; Dreisewerd, K.; Haier, J.; Senninger, N.; Friedrich, A. W.; Karch, H.; Hillenkamp, F.; Berkenkamp, S.; Peter-Katalinic, J.; Muthing, J., Matching IR-MALDI-o-TOF mass spectrometry with the TLC overlay binding assay and its clinical application for tracing tumor-associated glycosphingolipids in hepatocellular and pancreatic cancer. Anal Chem 2008, 80, (6), 1835-46.

20. Rohlfing, A.; Muthing, J.; Pohlentz, G.; Distler, U.; Peter-Katalinic, J.; Berkenkamp, S.; Dreisewerd, K., IR-MALDI-MS analysis of HPTLC-separated phospholipid mixtures directly from the TLC plate. Anal Chem 2007, 79, (15), 5793-808.

21. Bou Khalil, M.; Hou, W.; Zhou, H.; Elisma, F.; Swayne, L. A.; Blanchard, A. P.; Yao, Z.; Bennett, S. A.; Figeys, D., Lipidomics era: accomplishments and challenges. Mass Spectrom Rev 2010, 29, (6), 877-929.

22. Sullards, M. C.; Allegood, J. C.; Kelly, S.; Wang, E.; Haynes, C. A.; Park, H.; Chen, Y.; Merrill, A. H., Jr., Structure-specific, quantitative methods for analysis of sphingolipids by liquid chromatography-tandem mass spectrometry: "insideout" sphingolipidomics. Methods Enzymol 2007, 432, 83-115.

23. Shaner, R. L.; Allegood, J. C.; Park, H.; Wang, E.; Kelly, S.; Haynes, C. A.; Sullards, M. C.; Merrill, A. H., Jr., Quantitative analysis of sphingolipids for lipidomics using triple quadrupole and quadrupole linear ion trap mass spectrometers. J Lipid Res 2009, 50, (8), 1692-707.

24. Sommer, U.; Herscovitz, H.; Welty, F. K.; Costello, C. E., LC-MS-based method for the qualitative and quantitative analysis of complex lipid mixtures. $J$ Lipid Res 2006, 47, (4), 804-14.

25. Merrill, A. H., Jr.; Sullards, M. C.; Allegood, J. C.; Kelly, S.; Wang, E., Sphingolipidomics: high-throughput, structure-specific, and quantitative analysis of sphingolipids by liquid chromatography tandem mass spectrometry. Methods 2005, 36, (2), 207-24.

26. Veillon, L.; Go, S.; Matsuyama, W.; Suzuki, A.; Nagasaki, M.; Yatomi, Y.; Inokuchi, J., Identification of Ganglioside GM3 Molecular Species in Human Serum Associated with Risk Factors of Metabolic Syndrome. PLoS One 2015, 10, (6), e0129645.

27. Weishaupt, N.; Caughlin, S.; Yeung, K. K.; Whitehead, S. N., Differential Anatomical Expression of Ganglioside GM1 Species Containing d18:1 or d20:1 Sphingosine Detected by MALDI Imaging Mass Spectrometry in Mature Rat Brain. Front Neuroanat 2015, 9, 155. 
28. Nilsson, O.; Svennerholm, L., Accumulation of glucosylceramide and glucosylsphingosine (psychosine) in cerebrum and cerebellum in infantile and juvenile Gaucher disease. J Neurochem 1982, 39, (3), 709-18.

29. Hikita, T.; Tadano-Aritomi, K.; Iida-Tanaka, N.; Levery, S. B.; Ishizuka, I.; Hakomori, S., Cationic glycosphingolipids in neuronal tissues and their possible biological significance. Neurochem Res 2002, 27, (7-8), 575-81.

30. Jozwiak, W.; Koscielak, J., Lactosylsphingosine-reactive antibody and CEA in patients with colorectal cancer. Eur J Cancer Clin Oncol 1982, 18, (7), 617-21.

31. Neuenhofer, S.; Schwarzmann, G.; Egge, H.; Sandhoff, K., Synthesis of lysogangliosides. Biochemistry 1985, 24, (2), 525-532.

32. Song, X.; Ju, H.; Lasanajak, Y.; Kudelka, M. R.; Smith, D. F.; Cummings, R. D., Oxidative release of natural glycans for functional glycomics. Nat Methods 2016, $13,(6), 528-34$.

33. Hakomori, S. I., Release of carbohydrates from sphingoglycolipid by osmiumcatalyzed periodate oxidation followed by treatment with mild alkali. J Lipid Res 1966, 7, (6), 789-92.

34. Neville, D. C.; Coquard, V.; Priestman, D. A.; te Vruchte, D. J.; Sillence, D. J.; Dwek, R. A.; Platt, F. M.; Butters, T. D., Analysis of fluorescently labeled glycosphingolipid-derived oligosaccharides following ceramide glycanase digestion and anthranilic acid labeling. Anal Biochem 2004, 331, (2), 275-82.

35. Albrecht, S.; Vainauskas, S.; Stockmann, H.; McManus, C.; Taron, C. H.; Rudd, P. M., Comprehensive Profiling of Glycosphingolipid Glycans Using a Novel Broad Specificity Endoglycoceramidase in a High-Throughput Workflow. Anal. Chem. 2016, 88, (9), 4795-802.

36. Han, Y. B.; Wu, L.; Rich, J. R.; Huang, F. T.; Withers, S. G.; Feng, Y.; Yang, G. Y., Comprehensive characterization of sphingolipid ceramide N-deacylase for the synthesis and fatty acid remodeling of glycosphingolipids. Appl Microbiol Biotechnol 2015, 99, (16), 6715-26.

37. Keser, T.; Pavić, T.; Lauc, G.; Gornik, O., Comparison of 2-Aminobenzamide, Procainamide and RapiFluor-MS as Derivatizing Agents for High-Throughput HILIC-UPLC-FLR-MS N-glycan Analysis. Frontiers in Chemistry 2018, 6, (324).

38. Kushwah, D.; Patel, H. B.; Sinha, P. K.; Jana, P. K., Practical Approach for the Determination of Response Factors of Impurities in Drugs by HPLC. E-Journal of Chemistry 2011, 8, (4), 1504-1511.

39. Sturgill, E. R.; Aoki, K.; Lopez, P. H.; Colacurcio, D.; Vajn, K.; Lorenzini, I.; Majic, S.; Yang, W. H.; Heffer, M.; Tiemeyer, M.; Marth, J. D.; Schnaar, R. L., Biosynthesis of the major brain gangliosides GD1a and GT1b. Glycobiology 2012, 22, (10), 1289-301.

40. Lauber, M. A.; Yu, Y.-Q.; Brousmiche, D. W.; Hua, Z.; Koza, S. M.; Magnelli, P.; Guthrie, E.; Taron, C. H.; Fountain, K. J., Rapid Preparation of Released NGlycans for HILIC Analysis Using a Labeling Reagent that Facilitates Sensitive Fluorescence and ESI-MS Detection. Anal. Chem. 2015, 87, (10), 5401-5409.

41. Wing, D. R.; Garner, B.; Hunnam, V.; Reinkensmeier, G.; Andersson, U.; Harvey, D. J.; Dwek, R. A.; Platt, F. M.; Butters, T. D., High-performance liquid chromatography analysis of ganglioside carbohydrates at the picomole level after 
ceramide glycanase digestion and fluorescent labeling with 2-aminobenzamide. Anal Biochem 2001, 298, (2), 207-17.

42. Svennerholm, L.; Fredman, P., A procedure for the quantitative isolation of brain gangliosides. Biochim Biophys Acta 1980, 617, (1), 97-109.

43. Ulmer, C. Z.; Patterson, R. E.; Koelmel, J. P.; Garrett, T. J.; Yost, R. A., A Robust Lipidomics Workflow for Mammalian Cells, Plasma, and Tissue Using LiquidChromatography High-Resolution Tandem Mass Spectrometry. Methods Mol Biol 2017, 1609, 91-106.

44. Ulmer, C. Z.; Yost, R. A.; Chen, J.; Mathews, C. E.; Garrett, T. J., Liquid Chromatography-Mass Spectrometry Metabolic and Lipidomic Sample Preparation Workflow for Suspension-Cultured Mammalian Cells using Jurkat T lymphocyte Cells. J Proteomics Bioinform 2015, 8, (6), 126-132.

45. Novak, J.; Kriston-Pal, E.; Czibula, A.; Deak, M.; Kovacs, L.; Monostori, E.; Fajka-Boja, R., GM1 controlled lateral segregation of tyrosine kinase Lck predispose T-cells to cell-derived galectin-1-induced apoptosis. Mol Immunol 2014, 57, (2), 302-9.

46. Tu, J.; Yin, Y.; Xu, M.; Wang, R.; Zhu, Z.-J., Absolute quantitative lipidomics reveals lipidome-wide alterations in aging brain. Metabolomics 2017, 14, (1), 5.

47. Vieira, D. B.; Thur, K.; Sultana, S.; Priestman, D.; van der Spoel, A. C., Verification and refinement of cellular glycosphingolipid profiles using HPLC. Biochem Cell Biol 2015, 93, (6), 581-6.

48. Barrientos, R. C.; Zhang, Q., Isobaric Labeling of Intact Gangliosides toward Multiplexed LC-MS/MS-Based Quantitative Analysis. Anal Chem 2018, 90, (4), 2578-2586.

49. Hajek, R.; Jirasko, R.; Lisa, M.; Cifkova, E.; Holcapek, M., Hydrophilic Interaction Liquid Chromatography-Mass Spectrometry Characterization of Gangliosides in Biological Samples. Anal Chem 2017, 89, (22), 12425-12432.

50. Li, J.; Han, L.; Li, J.; Kitova, E. N.; Xiong, Z. J.; Prive, G. G.; Klassen, J. S., Detecting Protein-Glycolipid Interactions Using CaR-ESI-MS and Model Membranes: Comparison of Pre-loaded and Passively Loaded Picodiscs. $J$ Am Soc Mass Spectrom 2018, 29, (7), 1493-1504.

51. Kracun, I.; Rosner, H.; Drnovsek, V.; Heffer-Lauc, M.; Cosovic, C.; Lauc, G., Human brain gangliosides in development, aging and disease. Int J Dev Biol 1991, 35, (3), 289-95.

52. Posse de Chaves, E.; Sipione, S., Sphingolipids and gangliosides of the nervous system in membrane function and dysfunction. FEBS Lett 2010, 584, (9), 174859.

53. Lisa, M.; Holcapek, M., High-Throughput and Comprehensive Lipidomic Analysis Using Ultrahigh-Performance Supercritical Fluid Chromatography-Mass Spectrometry. Anal Chem 2015, 87, (14), 7187-95.

54. O'Brien, J. P.; Brodbelt, J. S., Structural characterization of gangliosides and glycolipids via ultraviolet photodissociation mass spectrometry. Anal Chem 2013, 85, (21), 10399-407.

55. Hirabayashi, Y.; Kimura, M.; Matsumoto, M.; Yamamoto, K.; Kadowaki, S.; Tochikura, T., A novel glycosphingolipid hydrolyzing enzyme, glycosphingolipid 
ceramide deacylase, which cleaves the linkage between the fatty acid and sphingosine base in glycosphingolipids. J Biochem 1988, 103, (1), 1-4.

56. Ito, M.; Kurita, T.; Kita, K., A novel enzyme that cleaves the N-acyl linkage of ceramides in various glycosphingolipids as well as sphingomyelin to produce their lyso forms. J Biol Chem 1995, 270, (41), 24370-4.

57. Daskhan, G. C.; Tran, H. T.; Meloncelli, P. J.; Lowary, T. L.; West, L. J.; Cairo, C. W., Construction of Multivalent Homo- and Heterofunctional ABO Blood Group Glycoconjugates Using a Trifunctional Linker Strategy. Bioconjug Chem 2018, 29, (2), 343-362.

58. Hori, A.; Yamashita, M.; Yamaura, M.; Hongo, M.; Honda, T.; Hidaka, H., Rapid quantitative analysis of human serum sphingomyelin species using MALDI-TOF mass spectrometry with lipid hydrolase treatment. Clin. Chim. Acta 2016, 453, 95-99.

59. Jenkins, B.; West, J. A.; Koulman, A., A Review of Odd-Chain Fatty Acid Metabolism and the Role of Pentadecanoic Acid (C15:0) and Heptadecanoic Acid (C17:0) in Health and Disease. Molecules 2015, 20, (2), 2425-2444.

60. Kita, K.; Kurita, T.; Ito, M., Characterization of the reversible nature of the reaction catalyzed by sphingolipid ceramide N-deacylase. A novel form of reverse hydrolysis reaction. Eur J Biochem 2001, 268, (3), 592-602.

61. Zama, K.; Hayashi, Y.; Ito, S.; Hirabayashi, Y.; Inoue, T.; Ohno, K.; Okino, N.; Ito, M., Simultaneous quantification of glucosylceramide and galactosylceramide by normal-phase HPLC using O-phtalaldehyde derivatives prepared with sphingolipid ceramide N-deacylase. Glycobiology 2009, 19, (7), 767-75.

62. Akino, T., Sphingosine base and fatty acid compositions of pig brain sphingolipids. Tohoku J Exp Med 1969, 98, (1), 87-97.

63. Caughlin, S.; Maheshwari, S.; Weishaupt, N.; Yeung, K. K.; Cechetto, D. F.; Whitehead, S. N., Age-dependent and regional heterogeneity in the long-chain base of A-series gangliosides observed in the rat brain using MALDI Imaging. Sci Rep 2017, 7, (1), 16135.

64. Fahy, E.; Subramaniam, S.; Brown, H. A.; Glass, C. K.; Merrill, A. H., Jr.; Murphy, R. C.; Raetz, C. R.; Russell, D. W.; Seyama, Y.; Shaw, W.; Shimizu, T.; Spener, F.; van Meer, G.; VanNieuwenhze, M. S.; White, S. H.; Witztum, J. L.; Dennis, E. A., A comprehensive classification system for lipids. J Lipid Res 2005, 46, (5), 839-61.

65. Schmelzer, K.; Fahy, E.; Subramaniam, S.; Dennis, E. A., The lipid maps initiative in lipidomics. Methods Enzymol 2007, 432, 171-83.

66. Tumanov, S.; Kamphorst, J. J., Recent advances in expanding the coverage of the lipidome. Curr Opin Biotechnol 2017, 43, 127-133. 